\title{
Possible Sources of the Difference between a Model Prediction and Observations of Bow Shock Crossings
}

\author{
Aleksey V. Dmitriev ${ }^{1}{ }^{*}$, Jih-Kwin Chao ${ }^{1}$, Ya-Hui Yang ${ }^{1}$, Ching-Huei Lin ${ }^{2}$, and De-Jin $\mathrm{Wu}^{3}$
}

(Manuscript received 14 March 2002, in final form 24 October 2002)

\begin{abstract}
The new data set of 1901 bow shock crossings by Geotail and Wind satellites in period 1999-2000 is selected for a wide range of distances and solar wind conditions observed by Wind and ACE upstream monitors. The data set is applied for verification of the semi-empirical bow shock model developed in the last solar minimum (1995-1997), using Geotail crossings of the bow shock and Wind upstream solar wind data. In general, the model accuracy is reasonably good in the dayside and flank regions of the bow shock. But, the model extrapolation in the tail region has much less accuracy due to restriction of the bow shock crossing distances used in the model development. Some difference between the data of ACE and Wind upstream solar wind monitors leads also to the model discrepancies. Analysis of long time variations of the bow shock parameters during last three solar cycles shows noticeable difference in the heliospheric conditions controlling the bow shock size and shape in the solar minimum and maximum. In the solar maximum, the variations of the interplanetary conditions are much higher than in the solar minimum. This difference produces additional disagreement in prediction of the bow shock crossings in the current solar maximum by the model developed using a data set obtained in the solar minimum.
\end{abstract}

(Key words: Bow Shock, Solar Wind Plasma, Interplanetary Magnetic Field, Solar Cycle)

\section{INTRODUCTION}

The bow shock (BS) is formed naturally as the supersonic solar wind collides with the Earth's magnetosphere obstacle. Therefore, the BS is very sensitive to the changing of the interplanetary conditions. The main parameter controlling the bow shock is the Mach number

\footnotetext{
${ }^{1}$ Institute of Space Science, National Central University, Chung-Li, Taiwan

${ }^{2}$ Department of Electrical Engineering, Nan-Jeon Institute of Technology, Yen-Shui, Taiwan

${ }^{3}$ Purple Mountain Observatory, Academia Sinica, Naking, China

${ }^{*}$ Corresponding author address. Dr. A. V. Dmitriev, Institute of Space Science, National Central University, Chung-Li, Taiwan 32054; E-mail: dalex@jupiter.ss.ncu.edu.tw
} 
(Spreiter et al. 1966). Due to the presence of the interplanetary magnetic field (IMF), the Mach number is considered a magnetosonic Mach number $(\mathrm{Mms})$. On the other hand, the size and shape of the bow shock depend on the size and shape of the magnetosphere obstacle (Spreiter and Stahara 1992; Verigin et al. 1997) controlled by solar wind dynamic pressure $P d$ and IMF $B z$ component. Many semi empirical models of the bow shock were developed on these suggestions (Farris and Russell 1994; Cairns and Lyon 1995; Verigin et al. 1997). The modern model by Chao et al. (2002) considers four upstream solar wind parameters for description of the bow shock size and shape: $M m s, P d, B z$ and thermal/magnetic pressure ratio $\beta$.

Comparison of the models and their verification by independent experimental data is usually based on study of case events. First efforts in this direction were devoted to a few observations of the extremely large size of the bow shock when the magnetosonic Mach number in the interplanetary medium approaches 1 . The comprehensive studies of the tenuous solar wind events (Richardson et al. 2000; Crooker et al. 2000) show that the occurrence probability of extremely low solar wind density varies significantly with solar cycle and becomes highest on the rising phase and in the solar maximum. The solar wind density is a very important component of the bow shock parameters $M m s, P d$, and $\beta$. But, these parameters depend also on other characteristics of the solar wind plasma and IMF. The solar cycle variations of the BS parameters have not been studied yet. But this is a very important step in the estimation of the model applicability for bow shocks under current solar wind conditions.

In the paper, we present the data set of the bow shock crossings provided by the Geotail and Wind satellites in the end of rising phase and maximum of the current solar cycle (19992000). ACE and Wind satellites are used as solar wind monitors producing the upstream solar wind conditions. We present the results of the bow shock model application for the new data set. The reasons of the prediction discrepancies of the model are discussed in the sense of different methods of data selection, calibration problems of upstream solar wind monitors and solar cycle variations of the bow shock parameters.

\section{BOW SHOCK LOCATION IN 1999-2000}

The bow shock locations in 1999-2000 are determined from its crossings by the Geotail and Wind satellites. The BS crossing is identified via plasma and magnetic field measurements. As a rule, in the magnetosheath the solar wind density and magnetic field strength are higher and solar wind velocity is smaller than in the interplanetary medium. But in the tail region some of the indicated differences may be very weak. Moreover, the experimental data sometimes have gaps or other quality problems leading to uncertainties in strictly determining the BS crossings.

An example of identification of the BS crossings by the Geotail (GE) satellite during 1213 May 2000 is presented in Fig. 1(a). The upstream solar wind conditions are obtained from the Wind (WI) satellite. The timing for the upstream data is corrected on solar wind propagation from the upstream monitor to the probe satellite. The top panel of Fig. 1 indicates the location of the Geotail (probe satellite) in GSE coordinate system in Earth radii (Re). The Xcoordinate is presented by solid line. The distance from the X-axis in the YZ plane (called $(\rho)$ is shown as a dotted line. The three next panels (from top to bottom) show the magnetic field 
GE \& WI: $12 / 05 / 2000$

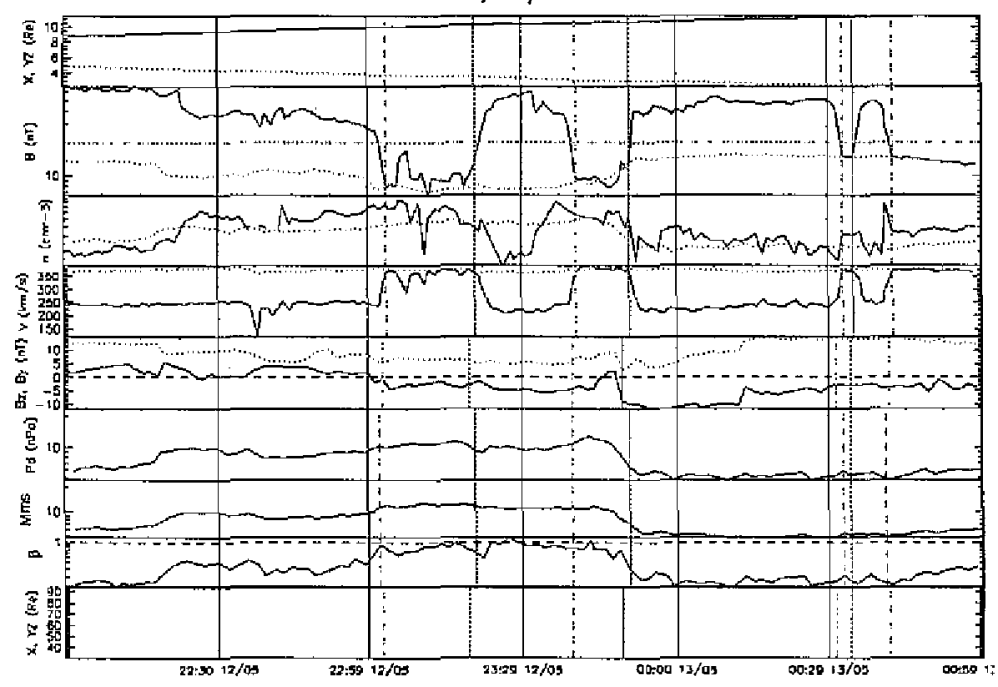

WI \& AC: $26 / 12 / 1999$

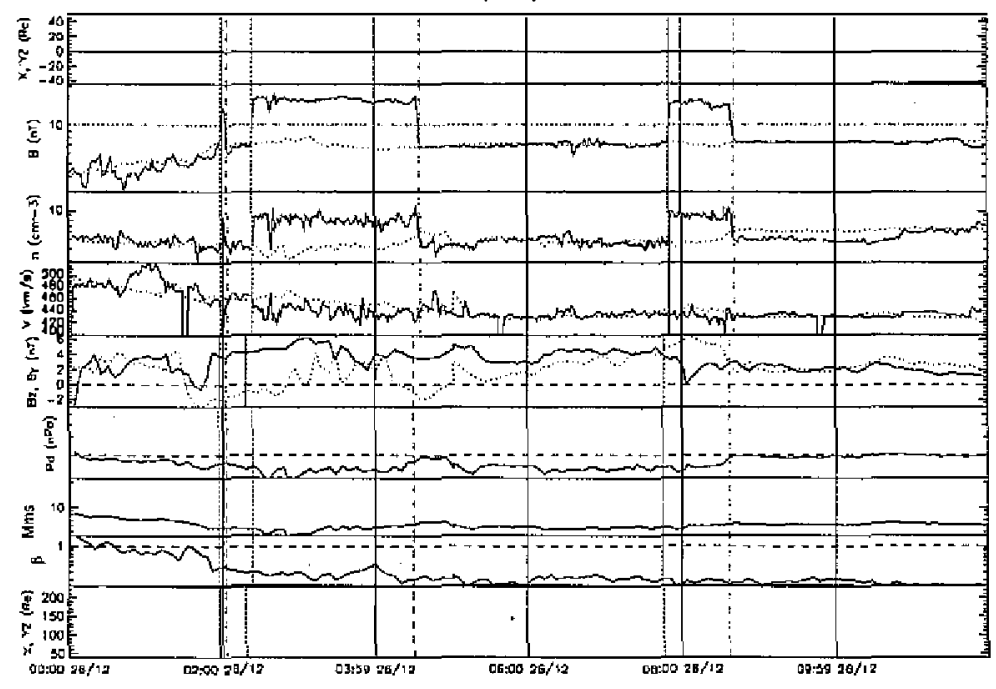

Fig. 1. Examples of identification of the bow shock crossings (vertical dashed and dotted lines) using observations of the Geotail (a) and the Wind (b) probe satellites (see the text for detail explanation).

magnitude $B$, plasma density $n$ and velocity $V$ measured by the Geotail (solid line) and by the Wind (dotted line). The next four panels present the parameters of the bow shock obtained from the Wind (upstream monitor) measurements of the upstream solar wind conditions: IMF $\mathrm{Bz}$ and $\mathrm{By}$ components (solid and dotted lines respectively), solar wind dynamic pressure $(P d)$, magnetosonic Mach number $(M m s)$ and thermal/magnetic pressure ratio $(\beta)$. The bottom panel shows locations of the Wind satellite in GSE co-ordinate system. The Wind is located at about $X=94 \operatorname{Re}$ toward the Sun and $\sim 29 \operatorname{Re}$ on the dawn. The Geotail is going toward 
the subsolar region. From 22:00 UT to $\sim 23: 00 \mathrm{UT}$, it is located in the magnetosheath where the magnetic field magnitude is several times higher than that one in the upstream region and the plasma velocity is much smaller ( $V \sim 250 \mathrm{~km} / \mathrm{s}$ ) relative to the upstream solar wind speed (V 350 $\mathrm{km} / \mathrm{s}$ ). It is interesting to note that the plasma density $\boldsymbol{n}$ in the magnetosheath does not differ from the solar wind density that may be connected with problems of the Geotail plasma data. At 23:02UT the Geotail crosses the bow shock (vertical dashed line) and enters interplanetary medium where it observes the plasma and IMF similar to the Wind measurements. This crossing is caused by gradual increase of the solar wind dynamic pressure $P d$, Mach number $M m s$ and $\beta$. At 23:20UT (vertical dotted line) the magnitudes of these parameters suddenly decrease and Geotail returns in the magnetosheath. The similar crossings are observed respectively at 23:40UT, 23:50UT and at 0:32UT, 0:34UT on May 13, 2000. At 0:41UT Geotail occurs far enough from the Earth and leaves the magnetosheath, entering the interplanetary space. In Fig. 1(a) we strictly identify 7 crossings of the dayside bow shock and therefore can estimate 7 different BS locations under different upstream solar wind conditions.

In Fig. 1(b) we present the example of the BS crossings by Wind (probe satellite) in the far tail region (X - -40Re) in December 26, 1999. The upstream solar wind conditions are measured by the ACE (AC) upstream monitor located near L1 point (X 230Re and $\rho \sim 40 \mathrm{Re}$ ). The Wind is going in the interplanetary medium along the geomagnetic tail at a distance YZ 50Re as indicated on the upper panel. As one can see from the three next panels the plasma and IMF observations on the probe satellite (solid curve) and on upstream monitor (doted curve) are in good agreement. The Wind satellite crosses the bow shock at 1:58UT, 2: 03UT, 2:23UT, 4:35UT, 7:50UT, and 8:40UT. These crossings are caused by changes of the solar wind dynamic pressure $P d$, Mach number $M m s$ and $\beta$. It is interesting to note that solar wind velocity in the far tail magnetosheath is the same as in the interplanetary medium but magnetosheath density and magnetic field are significantly higher.

The above described method reduces the error in determining the upstream solar wind conditions for bow shock crossings. This error originated mostly from incorrect determining of the time delay for radial propagation of the solar wind with inclined front from an upstream monitor to a probe satellite that is crossing the bow shock. The method allows varying the time delay for direct solar wind propagation to achieve maximal correlation between solar wind observations on upstream and on probe satellite. Therefore, we are able to determine the upstream solar wind conditions for the bow shock crossings with maximal possible accuracy restricted only by time resolution of the solar wind data. But, determining the distance to the bow shock may still have significant uncertainties due to large amplitude fluctuations of the bow shock relative to the probe satellite in transient events or due to large fluctuations in the solar wind.

This method of BS identification permits us to select 1362 BS crossings by the Geotail and 539 crossings by the Wind satellite. The crossings are presented in Fig. 2 in the $\{X, \rho\}$ GSE coordinate system where abscissa is X-coordinate and ordinate is the distance to the BS crossing from the $\mathrm{X}$ axis. The solid line indicates the average bow shock location. We can see that the X-coordinate of the Geotail crossings (Fig. 2a) is restricted by distance of about $23 \mathrm{Re}$ at the subsolar region and by $15 \mathrm{Re}$ toward the tail. The $\rho$-coordinate is restricted by a value of about $32 \mathrm{Re}$. Obviously, this restriction is explained by the relatively low apogee of the Geotail 


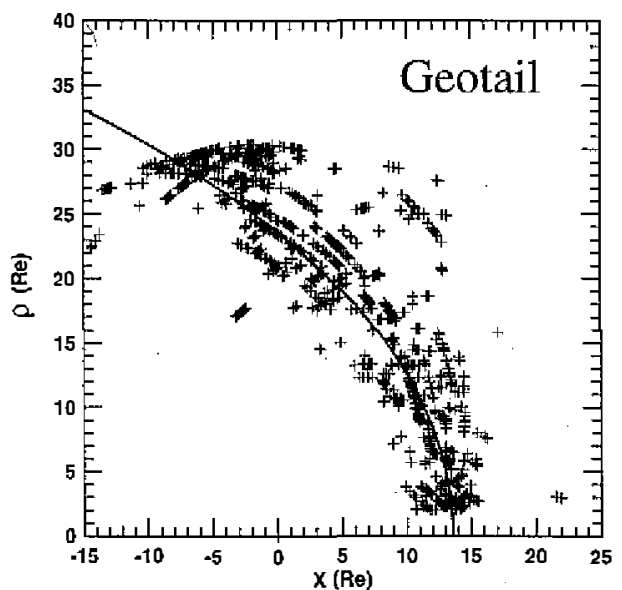

a

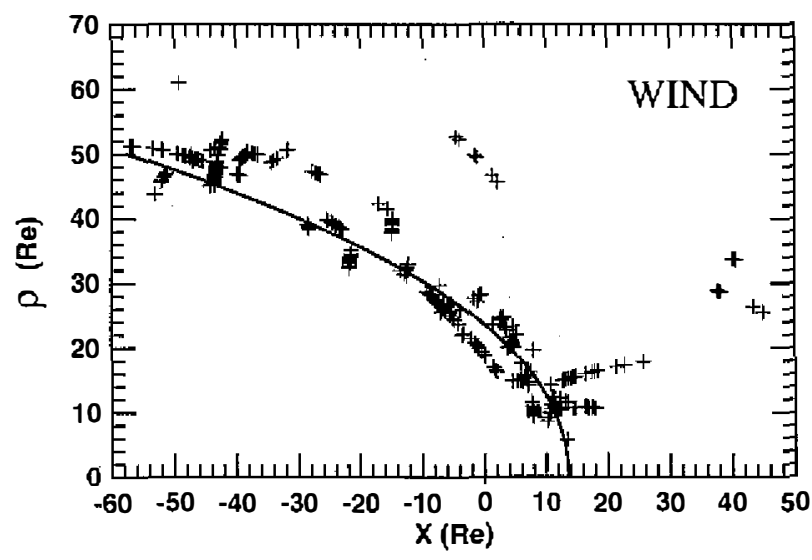

b

Fig. 2. Locations of the bow shock crossings (crosses) in the plane of revolution around the Sun-Earth axis detected by the Geotail (a) and the Wind (b) satellites in 1999 to 2000. Solid line is average BS location.

orbit. The Wind (Fig. 2 b) crossings have much larger spreadings due to higher apogee of the satellite. The crossings are observed in the range of X from -60 Re to $45 \mathrm{Re}$. The distance $\rho$ achieves $60 \mathrm{Re}$. On the other hand, the bow shock can be observed very close to Earth, at a distance of about $10 \mathrm{Re}$ on the dayside and $17 \mathrm{Re}$ on the flank. This wide range of bow shock locations is apparently associated with strong variability of the upstream solar wind conditions in the end of rising phase and maximum of the solar activity. Thus, the selected data set contains the BS crossings in a wide range of distances and upstream solar wind conditions that allow using it for comprehensive testing of the bow shock models.

\section{UPSTREAM CONDITIONS FOR THE BOW SHOCK CROSSINGS}

We separate the upstream solar wind conditions observed by the Wind and ACE upstream monitors. It is important to note here that, for the Geotail BS crossings, the upstream solar 
wind data may be obtained from the Wind or from the ACE satellite when the Wind data are not available or the Wind is not located in the interplanetary medium. For the Wind BS crossings, the upstream satellite is the ACE only. Therefore, the Wind upstream solar wind measurements correspond to BS crossings by the Geotail. The upstream solar wind conditions observed by the ACE are associated with either Geotail or Wind BS crossings.

The histograms of occurrence number distributions of the bow shock parameters measured by the Wind upstream solar wind monitor during the BS crossings by the Geotail satellite are presented in Fig. 3: a) for the solar wind dynamic pressure $P d$; b) for the Mach number $M m s ; c)$ for the thermal/magnetic pressure ratio $b$; and d) for the IMF $B z$-component. Figure 4 shows the same results as Fig. 3 for the measurements on the ACE upstream solar wind monitor during Wind and Geotail BS crossings. Gaussian fits of the histograms are presented by dotted curves. Parameters of the Gaussian fitting are indicated in the right top corner of the plots. Median, mean and most probable (Mpbl) values are presented in the left top corner of the plots and are indicated by respectively dashed, dashed dotted, and dotted vertical lines. The total number of points in a histogram is also indicated in the left top corner of the plots.

One can see clearly that all the BS parameters (excluding $B z$ ) have the log-normal distribution. Statistical distribution for the $B z$ is close to a normal shape in the linear scale. The main statistical moments of the parameters for Wind and ACE measurements are presented in Tables 1 and 2, respectively. It is important to note that the numbers of measurements performed by Wind and ACE upstream monitors for the bow shock crossings are practically the same (920 and 939 respectively). For both satellites, the most probable (Mpbl), median and mean values of the BS parameters are more or less equal. Both for the Wind and for the ACE measurements the solar wind dynamic pressure $P d$ has an average value of about $2 \mathrm{nPa}$, the average Mach number $M m s \sim 6.6$, the average thermal-magnetic pressure ratio $\beta \sim 0.7$ and the $B z$ average value is about $0 \mathrm{nT}$.

The dynamic ranges of the parameters measured by two different upstream solar wind monitors are quite different. The minimal and maximal values of the dynamic pressure observed by ACE satellite $(0.021 \mathrm{nPa}$ and $8.3 \mathrm{nPa}$ respectively) are several times less than those observed by the Wind satellite ( $0.16 \mathrm{nPa}$ and $26 \mathrm{nPa}$ respectively). The minimal value of Mach number observed by the ACE is also smaller than minimal Mach number observed by the Wind satellite ( 1.2 and 2 respectively). The significant difference is observed in the minimal value of thermal/magnetic pressure ratio. It is 3 times less for the ACE $(0.0065)$ relative to that obtained from the Wind satellite $(0.019)$. These differences may be explained partially by restriction of the Wind satellite apogee. Indeed, when the solar wind conditions become very weak ( $P d, M m s$ and $\beta$ have small values) the Wind satellite enters the magnetosheath and cannot be used as an upstream monitor.

To estimate an effect of the inter-calibration of upstream monitors, we compare statistical distribution of upstream solar wind parameters measured both by the ACE and by the Wind during all intervals of the Geotail BS crossings in 1999-2000 (see previous section). Figure 5 shows the statistical distribution of the upstream solar wind density $n$ (a) and IMF strength B (b) observed simultaneously by the Wind (solid histogram) and by the ACE (dashed gray histogram) during more than 900 hours when the BS crossings are identified using the Geotail data in 1999 to 2000 . The average values of the solar wind parameters measured by the ACE 

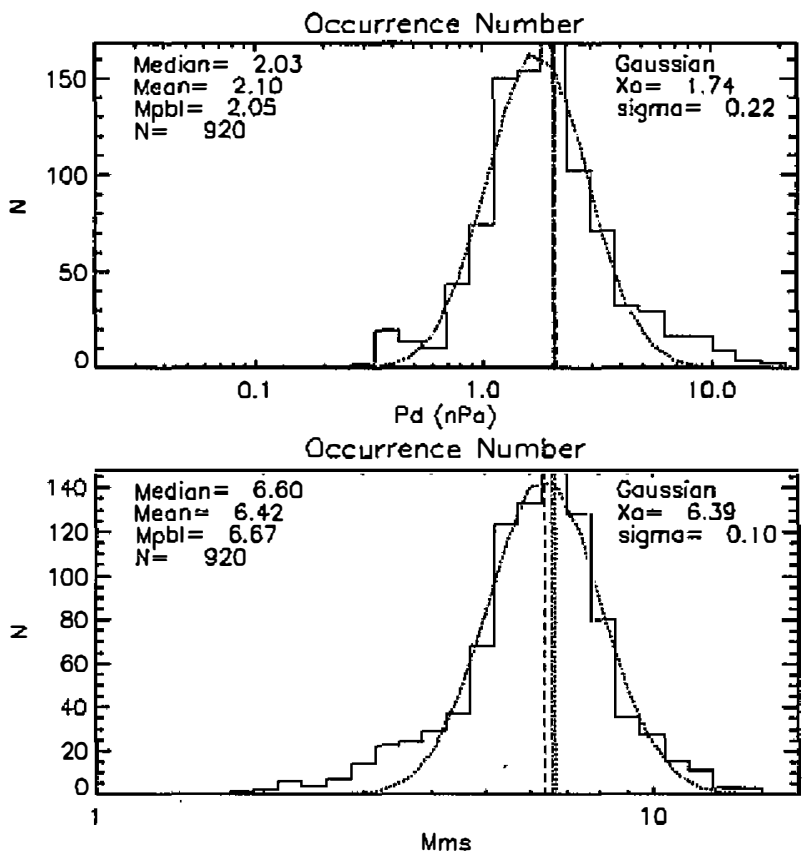

a

b
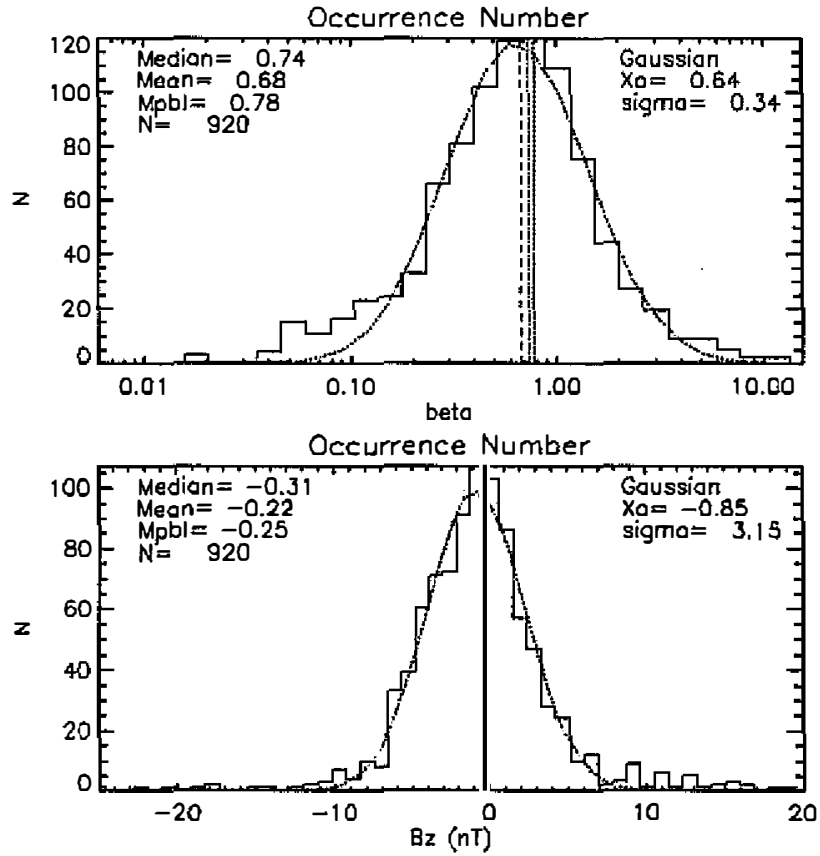

C

Fig. 3. Statistical distribution of the upstream solar wind conditions observed by Wind upstream monitor during BS crossings by the Geotail satellite in 1999 to 2000: dynamic pressure (a), magnetosonic Mach number (b), thermal/magnetic pressure ratio (c), and IMF $B z$-component (d) (see the text for detail explanation). 

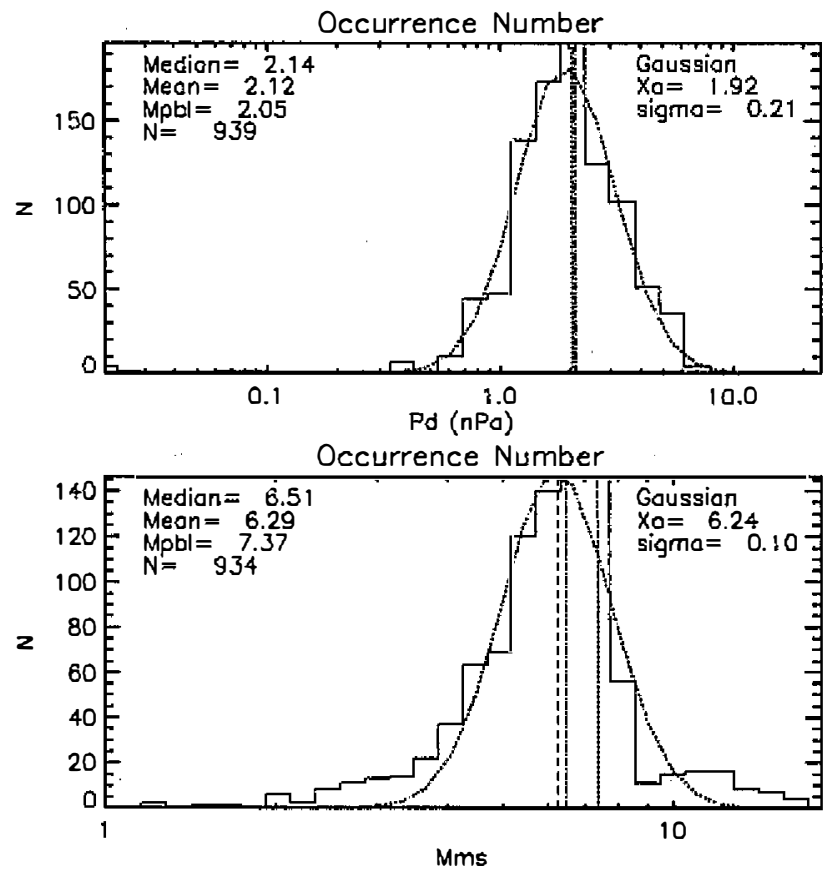

a

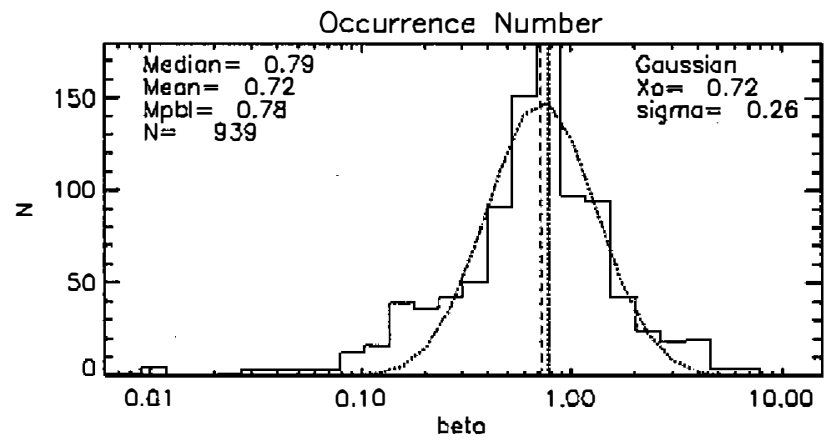

b

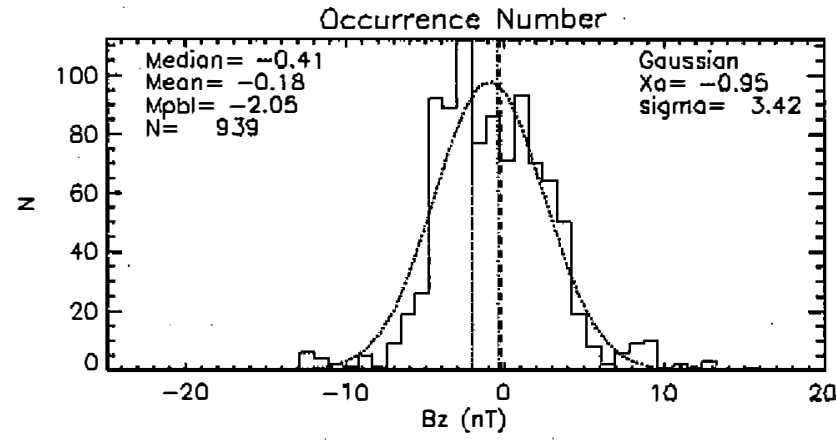

C

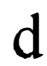

Fig. 4. Statistical distribution of the upstream solar wind conditions observed by ACE upstream monitor during BS crossings by the Geotail and Wind satellites in 1999-2000: dynamic pressure (a), magnetosonic Mach number (b), thermal/magnetic pressure ratio (c), and IMF Bz-component (d). 
Table 1. Main statistical properties of the bow shock parameters measured by Wind upstream monitor.

\begin{tabular}{|c|c|c|c|c|c|}
\hline . & Min & Max & Mpbl & Median & Mean \\
\hline $\mathrm{Pd}(\mathrm{nPa})$ & $616 \%$ & 26 & 2.0 & 2.0 & 2.1 \\
\hline Mms & (3. & 16. & 6.7 & 6.6 & 6.4 \\
\hline b & (1.4) & 19. & 0.72 & 0.74 & 0.68 \\
\hline $\mathrm{Bz}$ (nT) & -25. & 19. & -0.25 & -0.31 & -0.22 \\
\hline
\end{tabular}

Table 2. Main statistical properties of the bow shock parameters measured by ACE upstream monitor.

\begin{tabular}{|c|c|c|c|c|c|}
\hline & Min & $\operatorname{Max}$ & Mpbl & Median & Mean \\
\hline $\mathrm{Pd}(\mathrm{nPa})$ & $3 \% 10 \%$ & $18 \% \% \%$ & 2.1 & 2.1 & 2.1 \\
\hline Mms & - & 17 . & 7.4 & 6.5 & 6.3 \\
\hline $\mathrm{b}$ & $1 \% \% \%$ & 19. & 0.78 & 0.79 & 0.72 \\
\hline $\mathrm{Bz}(\mathrm{nT})$ & -12 & 16. & -2.1 & -0.4 & -0.18 \\
\hline
\end{tabular}

and Wind are close: 5.5 for density and 6.5 for IMF strength. But, the main parts of the distributions (in the vicinity of their maxima) are substantially different. One can see clearly that in many cases the ACE satellite measures smaller solar wind density and higher IMF strength than the Wind. This difference produces, as a rule, smaller values of the magnetosonic Much number calculated from the ACE data than that calculated from the Wind data. The thermal/ magnetic ratio $\beta$ calculated from the ACE data appears higher than the one calculated from the Wind data. Therefore, the problem of inter-calibration can cause disagreements in the bow shock model predictions based on upstream solar wind data produced by the ACE or the Wind satellites.

\section{COMPARISON WITH A BOW SHOCK MODEL}

To study the possible discrepancies between observations and model predictions, we apply the semi-empirical model of the bow shock by Chao et al. (2002) (CH02 model) for the BS crossings with associated upstream solar wind conditions measured by the Wind or the ACE satellites. The model describes the size and shape of the bow shock as functions of four input parameters: solar wind dynamic pressure $P d$, magnetosonic Mach number $\mathrm{Mms}$, thermal/magnetic pressure ratio $\beta$ and IMF $B z$-component in GSE coordinate system. For the model development 544 BS crossings by Geotail in 1995-1997 on radial distances from $12 \mathrm{Re}$ to $34 \mathrm{Re}$ 

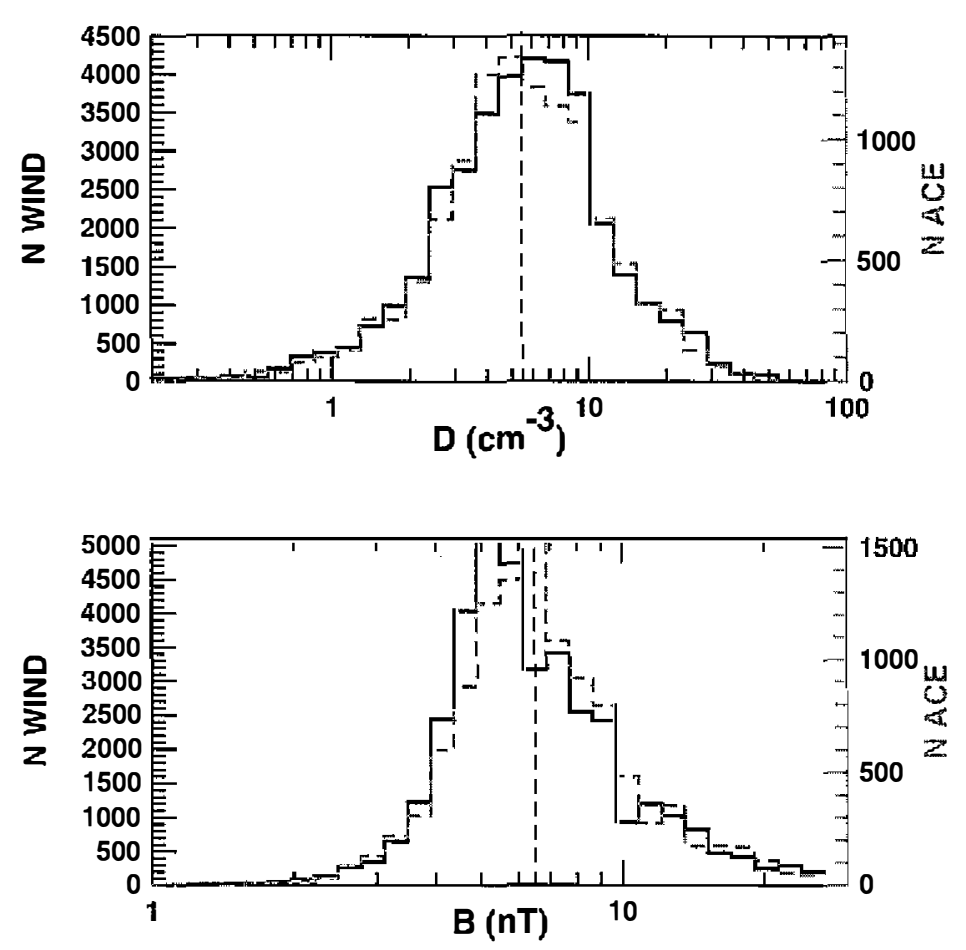

Fig. 5. Statistical distribution of the upstream solar wind density (a) and IMF strength (b) observed simultaneously by the Wind (solid histogram) and ACE (dashed gray histogram) satellites during intervals of the BS crossings observed by the Geotail in 1999 to 2000.

( $-5 \operatorname{Re}<\mathrm{X}<15 \operatorname{Re}, \rho<30 \operatorname{Re}$ ) with associated Wind upstream solar wind data were used. The data set contains only the BS crossings selected under quasi-steady upstream solar wind conditions when only one BS crossing is observed and occurs slowly, or multiple crossings are observed when values of the bow shock parameters change gradually. This method permits us to determine precisely the distance to the bow shock because its location under quasi-steady conditions is mostly close to the probe satellite in the crossing.

The model radial distance $\mathrm{R}$ of the bow shock at given cone angle $\theta$ is determined by Chao et al. (1999):

$$
R=r_{0}\left(\frac{1+\varepsilon}{1+\varepsilon \cos \theta}\right)^{\alpha},
$$

where the best-fit results for the parameters $r_{0}$ and ( can be expressed as the following two sets of equations, depending on the sign of $B z$ (Chao et al. 2002):

$$
\begin{aligned}
& \mathcal{E}=a_{12}, \\
& r_{0}=a_{1}\left(1+a_{2} B_{z}\right)\left(1+a_{9} \beta\right)\left(1+a_{4} \frac{\left(a_{8}-1\right) M_{m s}^{2}+2}{\left(a_{8}+1\right) M_{m s}^{2}}\right) P_{d}^{-1 / a_{11}} \text { for } B_{z} \geq 0,
\end{aligned}
$$




$$
\begin{aligned}
& \alpha=a_{5}\left(1+a_{13} B_{z}\right)\left(1+a_{7} D_{p}\right)\left[1+a_{10} \ln (1+\beta)\right]\left(1+a_{14} M_{m s}\right), \\
& r_{0}=a_{1}\left(1+a_{3} B_{z}\right)\left(1+a_{9} \beta\right)\left(1+a_{4} \frac{\left(a_{8}-1\right) M_{m s}^{2}+2}{\left(a_{8}+1\right) M_{m s}^{2}}\right) P_{\alpha}^{-1 / a_{11}} \text { for } B_{z}<0, \\
& \alpha=a_{5}\left(1+a_{6} B_{z}\right)\left(1+a_{7} D_{p}\right)\left[1+a_{10} \ln (1+\beta)\right]\left(1+a_{14} M_{m s}\right) .
\end{aligned}
$$

The expressions for $r_{0}$ are based on theoretical consideration of the bow shock formation (Spreiter et al. 1966). The term with solar wind dynamic pressure $P d$ is responsible for the magnetopause size and location as obstacle producing the bow shock. The complex term containing the magnetosonic Mach number $\mathrm{Mms}$ is also accepted from that theoretical investigation. Therefore, the $\mathrm{CH} 02$ semi-empirical model combines theoretical expressions with empirical presentation of the bow shock size and shape for the best approach to the experimental data. The coefficients $\boldsymbol{a}_{\mathrm{i}}$ are given in Table 3. This set of model coefficients produces the standard deviation of about $1.05 \mathrm{Re}$ on the model data set.

The results of the CH02 model application for the new data set in 1999-2000 are presented in Fig. 6. We estimate the model accuracy using two characteristics: standard deviation (SD) and relative error (RE). The standard deviation is calculated as:

$$
S D=\left\{\sum(R o b s-R)^{2} / N\right\}^{1 / 2},
$$

where Robs is the observed distance to the satellite crossing of the bow shock, $R$ is the model prediction of the BS crossing distance and $N$ is the number of crossings. The relative error is defined as:

$$
R E(\%)=100 \sum_{k=1}^{N}(|R o b s-R| / R o b s) / N .
$$

The relative error is useful for comparison of the model accuracy when the range of the model is very wide. In this case, even for a good model the $S D$ increases with distance but relative error does not change very much.

The standard deviation of the model prediction of the Geotail BS crossings based on the Wind upstream data in 1999-2000 (Fig. 6 a) equals $S D=3.2$ Re. The model accuracy for the predictions of the Geotail and Wind BS crossings based on the ACE upstream data (Fig. 6 b) is significantly smaller $(S D=11 \mathrm{Re})$. At the same time the standard deviation for the model data set in 1995-1997 is $S D=1.05$ (Chao et al. 2002). It is important to remember that the BS crossings based on the ACE upstream data have a very large dynamic range of distances (up to $80 \mathrm{Re}$ ). For the direct comparison, we indicate on the left side of Fig. 6 (b) the relative error

Table 3. Coefficients of the $\mathrm{CHO}$ bow shock model.

\begin{tabular}{lllll}
\hline$a_{1}=11.1266$ & $a_{2}=0.0010$ & $a_{3}=-0.0005$ & $a_{4}=2.5966$ & $a_{5}=0.8182$ \\
\hline$a_{6}=-0.0170$ & $a_{7}=-0.0122$ & $a_{8}=1.3007$ & $a_{9}=-0.0049$ & $a_{10}=-0.0328$ \\
\hline$a_{11}=6.047$ & $a_{12}=1.029$ & $a_{13}=0.0231$ & $a_{14}=-0.002$ &
\end{tabular}




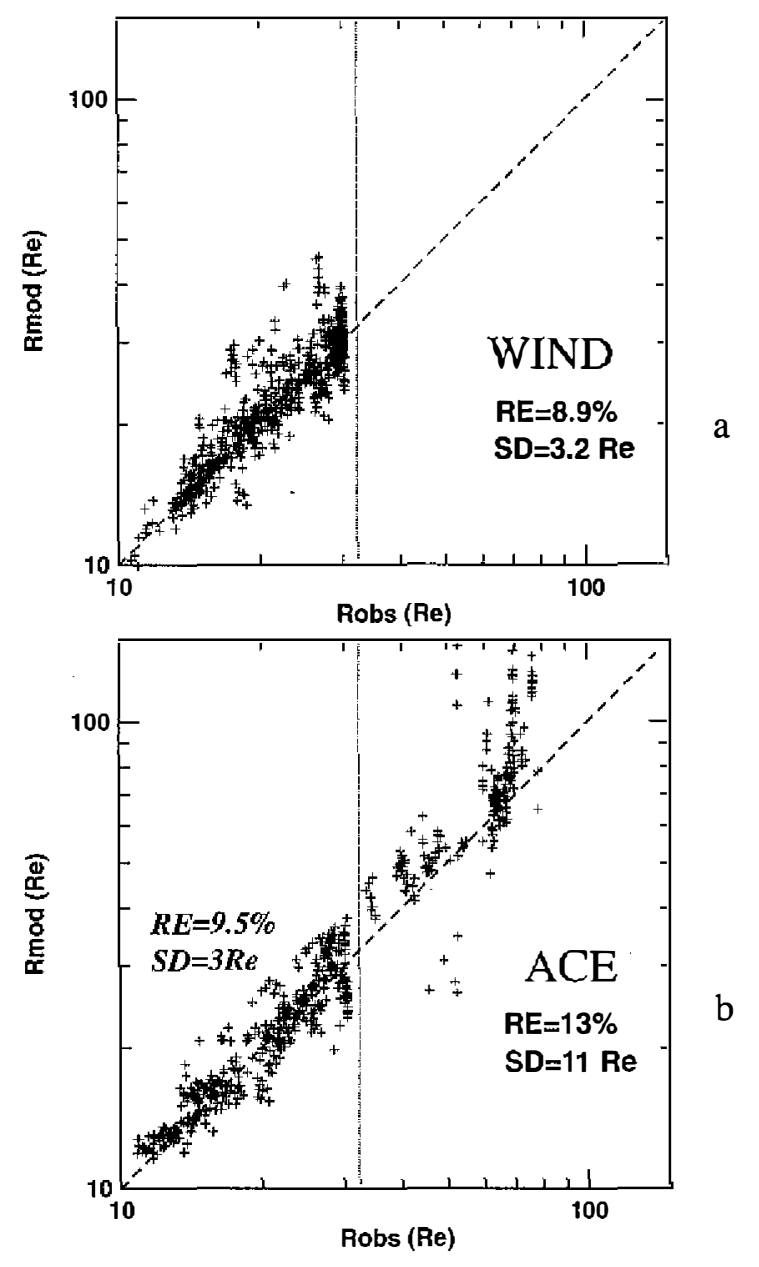

Fig. 6. Comparison of the Chao et al. (2002) bow shock model prediction with observations of the BS crossings for the Wind (a) and ACE (b) upstream solar wind monitors.

and standard deviation (italic font) for the ACE upstream data based model prediction of the BS crossings within the model dynamic range of the BS distances $(R o b s<34 \mathrm{Re})$. The distances of Geotail BS crossings with the Wind upstream monitor (Fig. 6 a) are just located inside the model dynamic range. We have to indicate here that the BS distances in the model dynamic range vary significantly from $\sim 10 \mathrm{Re}$ to $30 \mathrm{Re}$. In such a situation, the relative error $R E$ is a more reliable parameter for estimation of the model accuracy. Comparison of the model predictions based on the Wind and ACE upstream data shows that experimental data from the Wind produce better accuracy $(R E=8.9 \%)$ than data from the ACE $(R E=9.5 \%)$ despite on reverse ratio of the standard errors in this cases: $S D=3.2 \mathrm{Re}$ for the Wind and $\mathrm{SD}=3 \mathrm{Re}$ for the ACE upstream data. But, the standard deviation is inappropriate for estimation of the model accuracy in prediction of the widely varying function. Indeed, in Fig. 6 (a) the model prediction deviation increases with distance being relatively small for short BS distances. This behavior of the deviation is in good agreement with well known experimental fact that the BS fluctuations increase from the dayside where the BS distance is small to the flank and tail regions where the BS distances are much larger. The other dependence is observed in Fig. 6 
(b) where the model deviation does not change practically in the range of Robs from $13 \operatorname{Re}$ to 30 Re that indicate worse accuracy of the prediction at least for small and medium BS distances. Indeed the model errors at distances Robs $\sim 13 \mathrm{Re}$ and Robs $\sim 25 \mathrm{Re}$ are same and equal to about $5 \mathrm{Re}$. It means that on distances from $13 \mathrm{Re}$ to $25 \mathrm{Re}$ the deviation of the model prediction from the observations may exceed $20 \%$ of the absolute value. In the case of the Wind upstream monitor, the model prediction deviates much less for small distances (about $10 \%$ at Robs 13 $\mathrm{Re}$ ) than for large ones (about 50\% at Robs 30 Re). Further, we consider possible reasons of the discrepancies between the $\mathrm{CH} 02$ model prediction and observations of the BS crossings.

From Fig. 6, we can conclude that the largest errors of the model prediction are observed on large radial distances between 20 to $80 \mathrm{Re}$. As we indicated above, the CH02 model is developed in the range of the bow shock crossing at radial distances from $12 \mathrm{Re}$ to $34 \mathrm{Re}$. This fact may explain the increasing errors in the model ex rapolation to the large distances of the BS crossings observed mostly in the tail region. The additional reason for the $\mathrm{CH} 02$ model overestimation of the BS crossing distances in the vicinity of the Geotail apogee (about 30Re, see Fig. 6 a) and of the Wind apogee (about 70 Re, see Fig. 6 b) is the threshold effect. Indeed, when the bow shock shifts very far from the Earth, a probe satellite can not observe it at distances higher than its apogee. In this case, even correct model prediction does not often correspond to the observations because actually the equilibrium location of the bow shock is far from the probe satellite location. Therefore, for detailed study of model accuracy, we have to consider separately different regions of the bow shock.

The location of the bow shock crossings is divided into four regions: dayside $(X>5 \mathrm{Re})$, flank $(-5 \mathrm{Re}<\mathrm{X}<5 \mathrm{Re})$, close tail $(-35 \mathrm{Re}<\mathrm{X}<-5 \mathrm{Re})$ and far tail $(X<-35 \mathrm{Re})$. In each region, we estimate the standard deviation and relative error of the model predictions of the BS crossing distances. The results of the calculations are presented in Table 4. The first column indicates the bow shock region and the upstream solar wind monitor used for the model prediction of the BS crossings. The second and third columns show respectively the standard deviation SD and the relative error $R E$ of the model application. The number of the BS crossings $\mathrm{N}$ for each region is indicated in the fourth column.

As we can see from Table 4, the $\mathrm{CH} 02$ model has a good accuracy for prediction of the Geotail crossings using the Wind as upstream monitor for the dayside bow shock ( $\mathrm{SD}=2.1 R E$ and $R E=7.1 \%$ ). In the flank region the model error increases ( $\mathrm{SD}=3.1 \mathrm{Re}$ and $R E=9.4 \%)$ and in the close tail region the $\mathrm{CH} 02$ model extrapolation has less accuracy $(R E=12 \%)$. We have to remember that in the model data set the $\mathrm{CH} 02$ model has $\mathrm{SD}=1.05$ both for the dayside and for the flank region. Therefore, the model prediction is substantially different from the Geotail observations of the BS crossings in 1999-2000. We will continue to discuss this fact later.

In the case of the ACE upstream solar wind conditions for the Wind and the Geotail BS crossings the $\mathrm{CH} 02$ model has, in general, lower accuracy. On the dayside, the standard deviation and relative error are slightly higher than for the Wind upstream data: $S D=2.2 \mathrm{Re}$ and $R E=7.4 \%$. The errors increase rapidly in the flank $(R E=11 \%)$ and close tail $(R E=14 \%)$ regions and it dramatically increases in the far tail $(R E=19 \%)$. Therefore, we can conclude that BS crossings are predicted by $\mathrm{CHO} 2$ model with lower accuracy if the upstream solar wind conditions are determined from the ACE data. For the Wind upstream monitor the $\mathrm{CHO} 2$ model applicability is better. This fact may be explained by above discussed differences in the dy- 
Table 4. Accuracy of the bow shock model prediction of the BS crossings with upstream solar wind conditions observed by Wind and ACE satellites.

\begin{tabular}{|c|c|c|c|}
\hline & $\mathbf{S D}\left(\mathbf{R}_{\mathrm{E}}\right)$ & $\operatorname{RE}(\%)$ & $\mathbf{N}$ \\
\hline Model data set $15>X>-5 R_{E}$ & 1.05 & & 544 \\
\hline Wind $15>X>-5 R_{E}$ & 2.6 & 8.2 & 716 \\
\hline Wind $X>5 R_{E}$ & 2.1 & 7.1 & 384 \\
\hline Wind 5>X>-5 $R_{E}$ & 3.1 & 9.4 & 350 \\
\hline Wind $-5>X>-35 R_{E}$ & 5. & 12. & 170 \\
\hline ACE $15>X>-5 R_{E}$ & 3.2 & 9. & 542 \\
\hline ACE $X>5 R_{E}$ & 2.2 & 7.4 & 280 \\
\hline ACE $5>X>-5 R_{E}$ & 4.2 & 11. & 285 \\
\hline$A C E-5>X>-35 R_{E}$ & 6.3 & 14. & 171 \\
\hline ACE $X<-35 R_{E}$ & 20. & 19. & 197 \\
\hline
\end{tabular}

namic ranges of the BS parameters revealed from ACE and Wind observations and by possible problems with inter-calibration between these satellites. In the previous section, we have shown the differences for the solar wind dynamic pressure $P d$, Mach number Mms and ther$\mathrm{mal} / \mathrm{magnetic}$ pressure ratio $\beta$ obtained from the Wind and ACE data. This difference may lead to different model predictions based on the ACE and on the Wind upstream solar wind data. The problem of the upstream satellite inter-calibration is important especially under relatively quite solar wind conditions when the Wind satellite is located in the magnetosheath and only the ACE upstream data are available. Moreover, as we described above, the $\mathrm{CH} 02$ model was developed using only the Wind as upstream monitor. This means that the model error in prediction of the BS crossings using the ACE upstream data should be larger than error of the prediction based on the Wind data. Therefore, the inter-calibration of the solar wind monitors and uniform upstream solar wind data are very important in the development and testing of the bow shock models.

From the comparison analysis of the $\mathrm{CH} 02$ model accuracy in different regions, we find that its accuracy decreases rapidly from the dayside to the tail part of the bow shock. This fact is in agreement with the above discussed restriction of the ranges of the model data set. The model developed mostly in the dayside and on the flank region (X>-5 Re) may have incorrect extrapolation into the tail region $(X<-10 \mathrm{Re})$.

It is important to emphasize that the $\mathrm{CH} 02$ model predictions in the range $-5 \operatorname{Re}<\mathrm{X}<15$ Re for the new data set of the bow shock crossings in 1999 to 2000 have much lower accuracy 
than for the model data set of the BS crossings in 1995 to 1997. As we can see from the Table 4 , the model predictions in this region based on the Wind upstream solar wind data have standard deviation $S D=2.1 \mathrm{Re}$ that is twice as high as the standard deviation of the model data set prediction $(S D=1.05 \mathrm{Re})$. The model accuracy for the BS crossings based on the ACE upstream data is even less $(S D=3.2)$. Usually, this disagreement is explained by heterogeneity of the test data set of the BS crossings. Let us consider the nature of this heterogeneity.

The first reason of the heterogeneity originates from the difference of the data from different upstream solar wind monitors. This subject has been discussed above. The second reason is the difference in the methods used for selection of the model and new data sets. As we described above, the bow shock crossings from the $\mathrm{CH} 02$ model data set in 1995 to 1997 were selected under quasi-steady upstream solar wind conditions. In this case the probe satellite location during the BS crossings is very close to the bow shock. The method of the new data set selection is less restricted so that this data set includes practically any upstream conditions possible in 1999 to 2000. Apparently ransient events caused by solar wind sharp changes may produce bow shock locations strongly deviated from the probe satellite location that leads to "error" in the model prediction. Indeed, during such events the bow shock moves away from the satellite very rapidly and its location does not equal the satellite location. In this case, the model prediction of the bow shock distance may be significantly differ from the satellite one. Therefore, the method of the deviation (standard or relative) of the model prediction from the satellite location during BS crossings does not give adequate evaluation of the model accuracy. To avoid this problem, it is necessary to estimate the model quality in terms of the probability of prediction of the satellite location in the magnetosheath (Shue et al. 2000). Indeed, the qualitative determination of the satellite location in the magnetosheath may be successfully compared with the quantitative model prediction that the bow shock distance is greater or less than the radial distance of the probe satellite. This method is naturally used by any scientists who compare the model prediction with experimental data. In this case, the probability of the model prediction is simply calculated as relative number of events when the model correctly predicts the bow shock location relative to the probe satellite. This approach permits using any kind of data for independent estimation of the model accuracy without restriction on quasisteady upstream solar wind conditions. Application of this method for the model accuracy determination is subject of further work.

The other source of the heterogeneity is variations of the solar wind plasma and IMF parameters with the solar cycle. The period of 1995-1997 when the model data set was accumulated corresponds to the solar minimum. The new data set was selected in the end of rising phase and maximum of the current solar cycle (from 1999 to 2000). This difference in the solar activity leads to the difference in the upstream solar wind conditions controlling the bow shock.

\section{SOLAR CYCLE VARIATIONS OF THE BS PARAMETERS}

The long duration dynamics during 1963-2000 associated with solar cycle variations is presented in Fig. 7 for solar wind dynamic pressure $P d(a)$, magnetosonic Mach number $M m s$ (b), thermal/magnetic pressure ratio $\beta$ (c) and IMF $B z$ (d). The dynamics is represented in the 
form of floating histogram. The floating histogram contains 30 bins and it is accumulated during 4 months from hourly averaged values of the parameters. The time step between the histograms is 1 month. Color scale in the floating histogram indicates the relative occurrence number in logarithm scale as presented on color bar. Thick and thin black dotted curves and thin white dotted curves correspond to running average, one and three standard error deviations, respectively. The horizontal straight solid and dotted black lines indicate, respectively, the mean and $3 \sigma$-deviation for each parameter. As we mentioned above, all the bow shock parameters have log-normal statistical distribution (except $B z$ ). Therefore, the running average in the logarithmic scale should be close to the most probable value of the parameter. Figure 7 demonstrates it convincingly.

In Fig. 7 (a), one can see clearly the solar cycle variation of the running averaged and most probable values (black area) of the solar wind dynamic pressure $P d$ as well as the amplitude of the $P d$ fluctuations. In the solar minima $(1975,1985$ and 1996), the average and most probable dynamic pressure are higher than in the maxima $(1970,1980,1991,2000)$. For example, the running average $P d$ in 1996 and in 2000 is equal to about $P d=2.5 \mathrm{nPa}$ and $P d=2.0 \mathrm{nPa}$, respectively. The amplitude of the variations increases also in the solar maxima when the hourly averaged pressure may decrease down to very low values $(P d<0.1 \mathrm{nPa})$ and increase to $100 \mathrm{nPa}$. In the solar minima, the statistical distribution of $P d$ is much narrower than in maxima.

The Mach number Mms (Fig. $7 \mathrm{~b}$ ) anti-correlates with solar activity. In the solar maxima, the occurrence probability of low Mach number is higher than in the solar minima when the relatively large $M m s$ values predominate. The ratio between the running average $M m s$ value in the last solar minimum (Mms 10 in 1996) and in the current maximum (Mms 6.3 in 2000) is more than 1.5. The thermal/magnetic pressure ratio $\beta$ (Fig. $7 \mathrm{c}$ ) has the same dynamics with the solar cycle and the same amplitude of its solar cycle variation.

The average value of the IMF $B z$ (Fig. 7 d) does not change with solar cycle and lays in the vicinity of $0 \mathrm{nT}$. But, the $B z$ variations in the solar maxima are about twice as high as in the minima. This fact is in good agreement with the increase of the solar wind disturbances during high solar activity.

Analysis of the Fig. 7 shows that the bow shock parameters vary substantially with solar cycle. The $\mathrm{CH} 02$ model was developed from the data set of the bow shock crossings in 19951997 i.e., in the last solar minimum. The new data set is selected in the vicinity of the solar maximum (1999-2000) when the average values of the solar wind dynamic pressure, Mach number and thermal/magnetic pressure ratio are smaller than in the solar minimum. Moreover, in the solar maximum extremely high/low values of the BS parameters occur more often. In other words, the $\mathrm{CH} 02$ model should have higher accuracy for prediction of the bow shock crossings under relatively higher dynamic pressure, Mach number and $\beta$ and in moderate dynamic range of the BS parameters.

To demonstrate this suggestion, we present in Fig. 8 two case events of the $\mathrm{CH} 02$ model prediction of the bow shock location in comparison with the Geotail observations of the BS crossings. The upstream solar wind conditions are obtained from the Wind satellite. The model prediction of the bow shock location under strong solar wind dynamic pressure is shown on the top panel of Fig. 8 (a) by solid curve. The Geotail radial distance is presented in the top panel by smoothed solid line. The shadow bars indicate time intervals when the Geotail is 


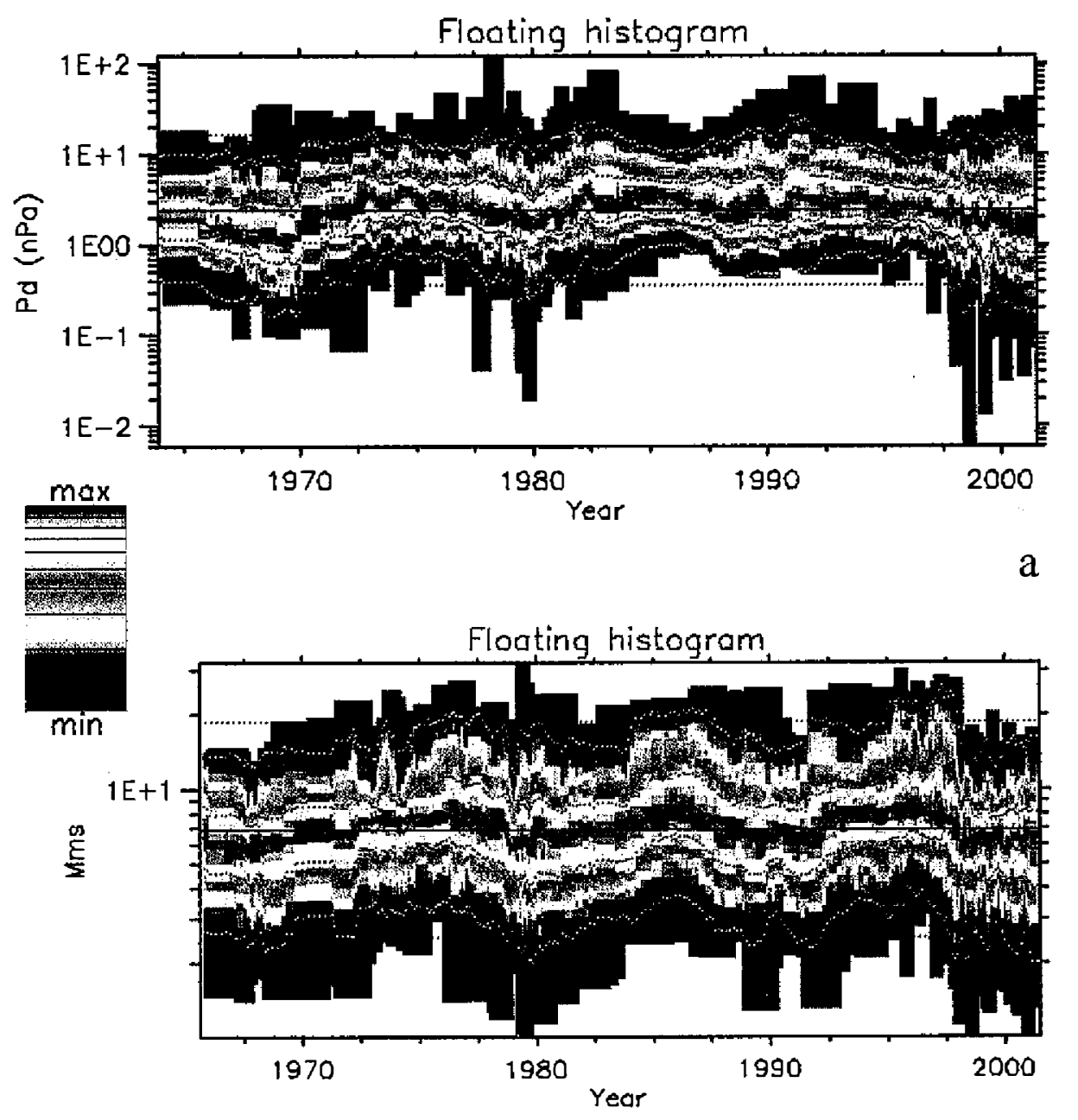

b

Fig. 7. Floating histograms characterizing the solar cycle variations of the solar wind dynamic pressure (a), magnetosonic Mach number (b), thermal/ magnetic pressure ratio (c) and IMF $B z$. (d). The horizontal solid and dotted black lines are the mean and $3 \sigma$ deviation of the corresponding parameter. The thick and thin black dotted curves are running average and 1( deviation of the corresponding parameter. See the text for more comments.

located in the interplanetary medium. These intervals are identified from the magnetic field measurements by the Geotail probe satellite and by the Wind upstream monitor presented on the second panel (from top to bottom) by solid and dotted curves, respectively. During these intervals, the Geotail magnetic field measurements are very similar to the Wind measurements in the interplanetary medium. However, when the Geotail is located in the magnetosheath, it measures a much stronger magnetic field than the IMF. Vertical solid lines indicate Geotail 


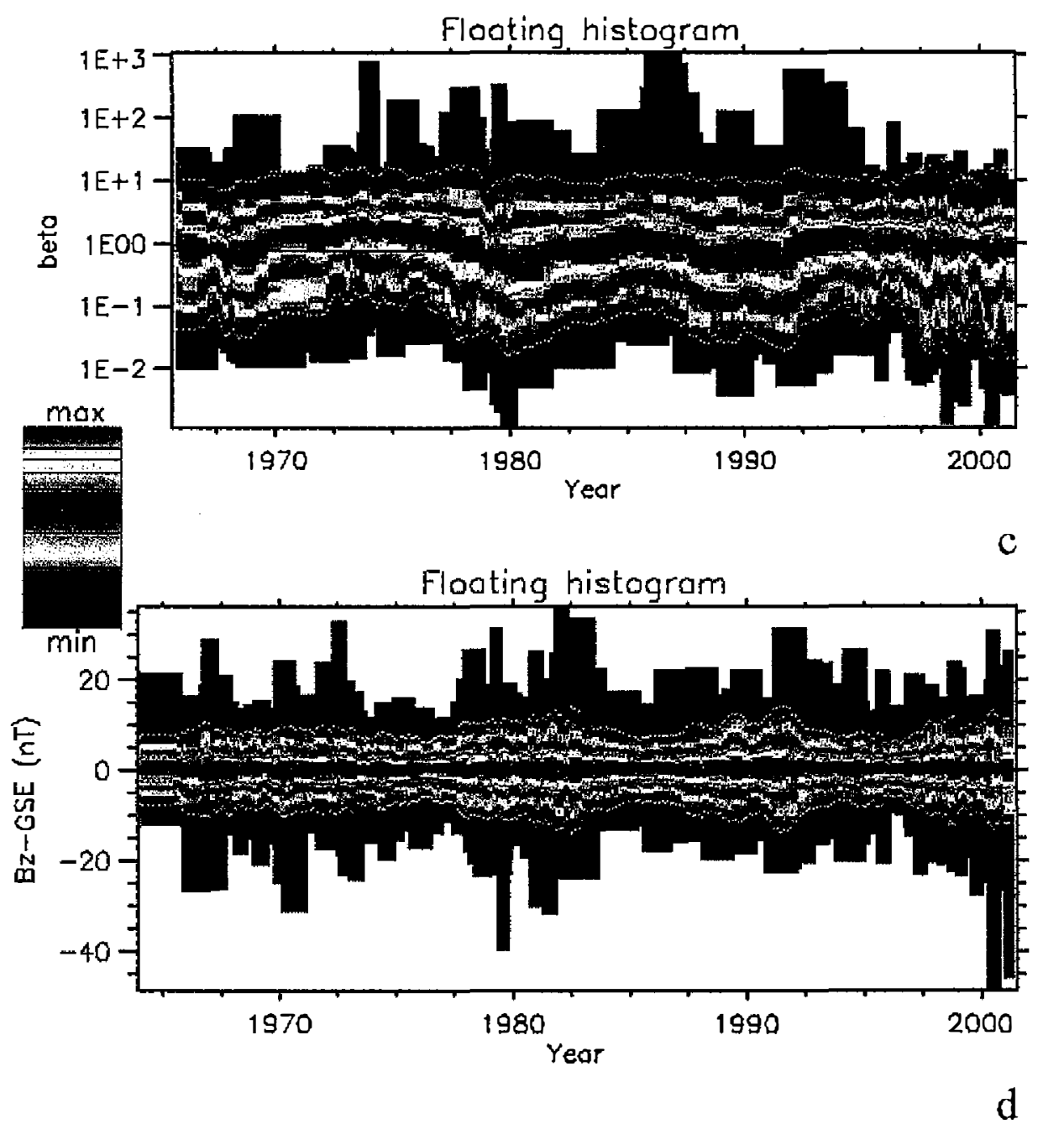

(Fig. 7. continued)

entrances in the magnetosheath when magnetic field measured by Geotail sudden increases. Vertical dashed lines indicate BS crossings when Geotail comes out to the interplanetary medium and begins to measure the same magnetic field as the Wind upstream monitor. The next three panels show bow shock parameters and upstream solar wind conditions: solar wind dynamic pressure $P \boldsymbol{d}$, Mach number $M m s$ (solid curve) and thermal/magnetic pressure ratio $\beta$ (dotted curve) as well as IMF $B z$ (solid line) and $B y$ (dotted line) components in GSE coordinate system (bottom panel).

On 6-7 April 2000 (Fig. 8a) the Geotail goes from the dayside (at 16:00UT) to the flank (after 00UT on 7 April) and observes multiple bow shock crossings associated with the arrival of an interplanetary shock at $\sim 17: 00 \mathrm{UT}$ on April 6 . The $\mathrm{CH} 02$ model predicts BS crossings very well when the Geotail is located on the dayside (16UT-18UT). But, after 20UT when the Geotail approaches to the flank region the model predicts several false BS crossings due to 

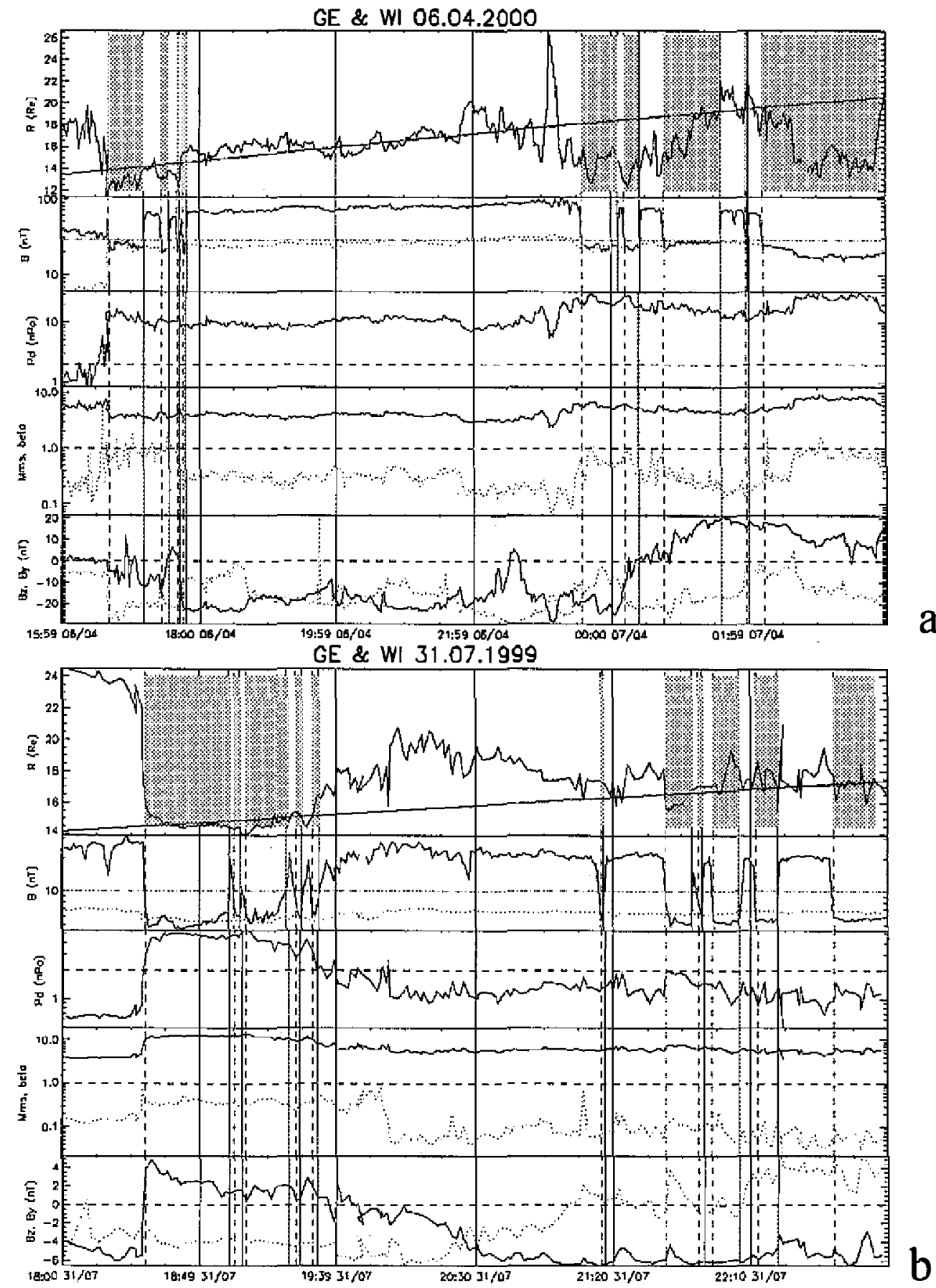

Fig. 8. Comparison of the $\mathrm{CHO} 2$ model prediction of the BS location based on the Wind upstream solar wind data with the Geotail probe satellite observations (see the text for detail explanation). 
overestimation of the bow shock compression. The overestimation is most prominent in the - time period from 22UT on April 6 to 2UT on April 7 when the solar wind dynamic pressure and the southward IMF component are relatively high (i.e., $P d \sim 20 \mathrm{nPa}$ and $B z \sim-20 \mathrm{nT}$ ) and the Mach number is relatively low $M m s \sim 3$ to 5 .

On July 31, 1999 (Fig. 8b), the Geotail satellite is located on the dayside. The solar wind dynamic pressure is moderate or even small during this time. Weak interplanetary shock with $P d \sim 4 \mathrm{nPa}$ arrives to the Geoatil at 18:30UT and pushes the bow shock inside the Geotail orbit. But, the $\mathrm{CHO2}$ model predicts this crossing with some delay when solar wind dynamic pressure becomes higher than $4 \mathrm{nPa}$. During the time interval from 18:30UT to 19:30UT, the model predicts strong bow shock compression and misses a few BS crossings detected by the Geotail. After 22UT when both solar wind dynamic pressure and thermal/magnetic pressure ratio become relatively small $(P d \sim 1 \mathrm{nPa}$ and $\beta<0.1)$, the model prediction is practically incorrect and anti correlates with bow shock location detected by the Geotail satellite, namely, when the Geotail is located in the interplanetary medium the model predicts magnetosheath interval and vise versa.

Therefore from the two examples we can see clearly that the $\mathrm{CHO2}$ model capability decreases in predictions of the BS crossings under some "unusual" solar wind conditions when the bow shock parameters have very large or small values. Such conditions predominate just in the vicinity of and during solar maxima when the interplanetary medium is strongly disturbed by high solar activity.

To demonstrate additional contributions to discrepancies of the model prediction originating from the difference of upstream solar wind data sources, we present in Fig. 9 the $\mathrm{CHO} 2$ model prediction of the BS location based on the ACE upstream data for the same time intervals as presented in Fig. 8. The probe satellite for the identification of the BS crossings is the Geotale. The comparison of Figs. 8 and 9 shows that the model prediction based on the ACE upstream data gives mostly smaller BS distances than the model prediction based on the Wind data, especially during the time intervals from 00:00UT to 4:00UT on April 7 and from 18: 30UT to 19:30UT on July 31. In these two case events the use of the ACE upstream data leads to systematical model underestimation of the BS location, and therefore produces additional discrepancies between the model prediction and observations of the bow shock crossings.

\section{DISCUSSION AND CONCLUSIONS}

The CH02 model application for prediction of the bow shock crossings in 1999-2000 shows a disagreement between the model predictions and observations. We have considered a few possible reasons for the disagreement. The most significant source of the model errors is the method of the new data set selection, which is unable to exclude cases of transient events and of high amplitude fluctuations of the bow shock. Indeed, for these cases, uncertainties in the distance to the bow shock are very big because actual location of the bow shock is significantly different from the probe satellite position, which is assumed equal to the bow chock location.

Thus, even if the new data set will be improved by additional selection on the quasisteady upstream solar wind conditions, the model prediction will still produce disagreement 

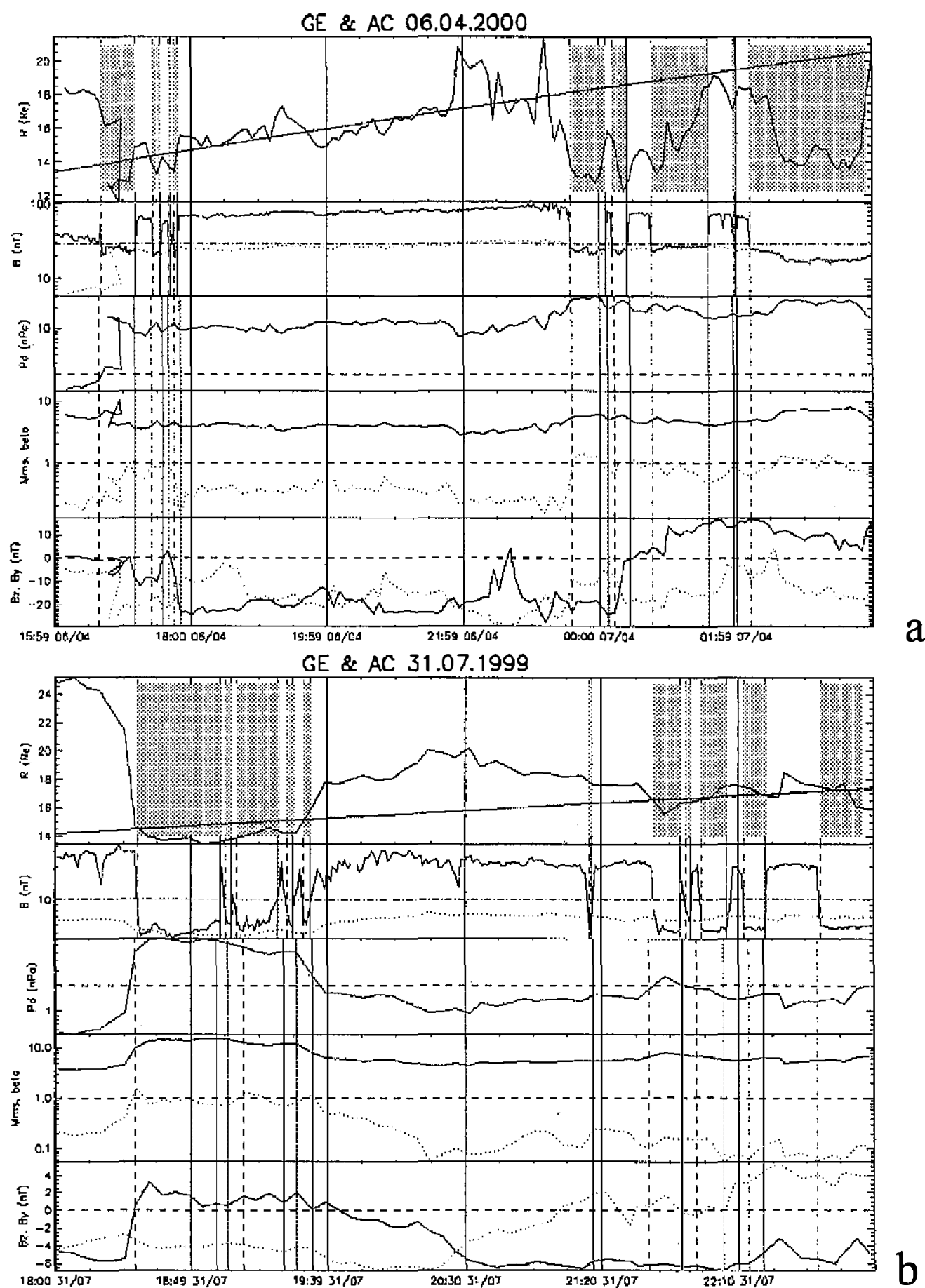

Fig. 9. Same as on Fig. 8, but for the ACE upstream solar wind data. 
originating from the other sources. Namely, there are significant differences in the dynamic ranges of the model and new data set expanded far into the tail region, problems with intercalibration of the upstream monitors, and solar cycle variations of the bow shock parameters. These factors necessarily become sources of disagreements in application of the method of the probability of prediction of the probe satellite location relative to the bow shock. Therefore, we can give the following sources independent of the method of data selection and responsible for the disagreement between the bow shock model prediction and observations of the BS crossings.

1. The ACE upstream solar wind data are slightly different from the data produced by the Wind satellite partially due to problems in the inter-calibration of their plasma and magnetic field detectors. This difference explains the decrease of the model accuracy in the prediction of the bow shock location based on the upstream data produced by the ACE satellite because the model was developed using only Wind upstream data.

2. The discrepancies between the bow shock locations observed by the Geotail and predicted by the model from the Wind upstream solar wind data are caused by two reasons. The dynamic range of the model is restricted by the Geotail crossings of the bow shock in the dayside and close tail region. Firstly, the model extrapolation into far tail may have substantial deviation from the actual bow shock location. Secondly, the model based on the data obtained in the solar minimum (1995-1997) better predicts the bow shock crossings under moderate level of values of the solar wind dynamic pressure, magnetosonic Mach number and thermal/magnetic pressure ratio. At the end of the rising phase and maximum of the solar activity in 1999 to 2000, the bow shock parameters have in general smaller average values and their variations are significantly higher than in the solar minimum. Thereby, contrary to the solar minimum, during the considered period of 1999 to 2000 there are many events that require an extrapolation for the empirical model. These events are associated with either extremely quiet interplanetary conditions when the bow shock parameters have unusually small values or strong interplanetary disturbances characterized by extremely high solar wind dynamic pressure and/or negative IMF $B z$.

Acknowledgement This work is supported by grants NSC-90-2811-M-008-010 and NSC90-2111-M-232-001 from the National Science Council.

\section{REFERENCES}

Cairns, I. H., and J. G. Lyon, 1995: MHD simulations of Earth's bow shock at low Mach numbers: Standoff distance. J. Geophys. Res., 100, 17,173-17,180.

Chao, J. K., C.-H. Lin, D. J. Wu, M. Kessel, S. H. Chen, and R. Lepping, 1999: A model for the size and shape of the Earth's bow shock, EOS, Transactions, AGU 1999 Fall Meeting, Vol. 80, No. 46, F897.

Chao, J. K., D. J. Wu, C.-H. Lin, Y. H. Yang, and X. Y. Wang, 2002: Models for the size and shape of the Earth's magnetopause and bow shock, Space Weather Study Using Multipoint Techniques, Ed. L.-H. Lyu, Pergamon, 360p.

Crooker N. U., S. Shodhan, J. T. Gosling, J. Simmerer, R. P. Lepping et al. 2000: Density 
extremes in the solar wind. Jeophys. Res. Lett., 27, 23, 3769-3772.

Farris, M. H., and C. T. Russell, 1994: Determining the standoff distance of the bow shock: Much number dependence and use of models. J. Geophys. Res., 99, 17,681-17,689,

Richardson I. G., D. Berdichevsky, M. D. Desch, C. J. Farrigua, 2000: Solar cycle variation of low density solar wind during more than three solar cycles. Jeophys. Res. Lett., 27, 23, 3761-3764.

Shue J.-H., P. Song, C. T. Russell, J. K. Chao, and Y.-H. Yang, 2000: Toward predicting the position of the magnetopause within geosynchronous orbit. J. Geophys. Res., 105, A2, 2641-2656.

Spreiter, J. R., and S. S. Stahara, 1992: Computer modeling of solar wind interaction with Venus and Mars, In Venus and Mars: Atmospheres, Ionospheres and Solar Wind Interactions. Geophys. Monogr. Ser., vol. 66, edited by J.H. Luhmann, M. Tatrallyay, and R.O. Pepin, pp. 345, AGU, Washington, D.C.

Spreiter, J. R., A. L. Summers, and A. Y. Alksne, 1966: Hydromagnetic flow around the magnetoshere. Planet. Space Sci., 14, 223-238.

Verigin, M. I., G. A. Kotova, A. P. Remizov, N. M. Shutte, K. Schwingenschuh, W. Riedler, T. L. Zhang, H. Rosenbauer, K. Szego, M. Tatrallysy, and V. Styazhkin, 1997: Studies of the Martian bow shock response to the variation of the magnetosphere dimensions according to TAUS and MAGMA measurements aboard the Phobos 2 Orbiter. Adv. Space Res., 20, No 2, 155-159. 